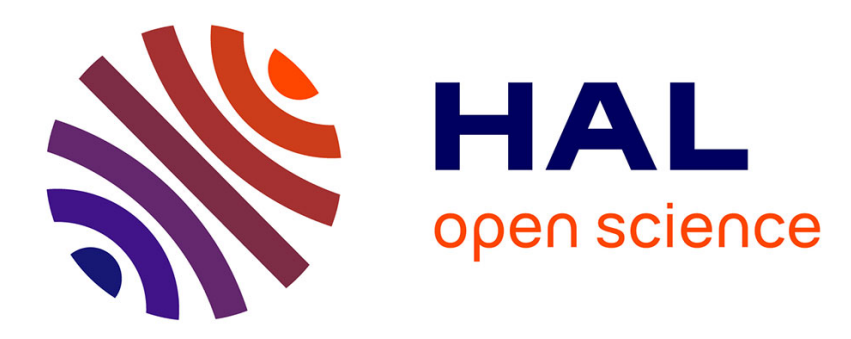

\title{
Une épiphanie de Zeus Sôter à Clazomènes
}

Thibaut Boulay

\section{To cite this version:}

Thibaut Boulay. Une épiphanie de Zeus Sôter à Clazomènes. Revue Numismatique, 2009, 6 (165), pp.113-127. 10.3406/numi.2009.2870 . hal-01262603

\section{HAL Id: hal-01262603 https://hal.science/hal-01262603}

Submitted on 26 Jan 2016

HAL is a multi-disciplinary open access archive for the deposit and dissemination of scientific research documents, whether they are published or not. The documents may come from teaching and research institutions in France or abroad, or from public or private research centers.
L'archive ouverte pluridisciplinaire HAL, est destinée au dépôt et à la diffusion de documents scientifiques de niveau recherche, publiés ou non, émanant des établissements d'enseignement et de recherche français ou étrangers, des laboratoires publics ou privés. 


\title{
Une épiphanie de Zeus Sôter à Clazomènes
}

\section{Thibaut Boulay}

\section{Résumé}

Les émissions, par Clazomènes, de tétradrachmes à types personnels au milieu du lle s. av. J.-C. figurant au droit Zeus Sôter Épiphanès et au revers une amazone ne peuvent être mises en relation avec la victoire d'Eumène II sur les Galates en 166. Ces monnaies, frappées à l'occasion de panégyries, célébraient une épiphanie salvatrice de Zeus Sôter, peut-être au cours de la guerre qui opposa Prusias II à Attale II.

\begin{abstract}
Summary - Small hoards buried north of the frontiers of the kingdom of Macedonia that contain heavy tetrobols of Perdikkas II, of the Chalcidic League and Acanthus may be related with the military operations of Brasidas and his allies, among which the Macedonian king, the Chalcideans of Thrace and Acanthus, against Arrhabaeus of Lynkos.
\end{abstract}

\section{Citer ce document / Cite this document :}

Boulay Thibaut. Une épiphanie de Zeus Sôter à Clazomènes. In: Revue numismatique, 6e série - Tome 165, année 2009 pp. 113-127.

doi : 10.3406/numi.2009.2870

http://www.persee.fr/doc/numi_0484-8942_2009_num_6_165_2870

Document généré le 06/01/2016 


\section{Thibaut $\mathrm{B}()(1,1) \%$ \\ Une épiphanie de Zeus Sôter à Clazomènes'}

Résumé - I es émissions, par Claromènes, de tétradrachmes à types personnels au milieu du 1" s. av. J.-C. figurant au droit \%eus Sôter Épiphanès et au revers une amazone ne peuvent être mises en relation avec la victoire d'Eumène 11 sur les Galates en 166. Ces momnaies. frappées a l'occassion de panégyries. célébraient une épiphanie salvattrice de \%eus Sôter peut-être au cours de la guerre qui opposa Prusiass II à Attale II.

Summary - Autonomous tetradrachms issued by Clayomenes in the mid-second century B.C. in the name and types of \%eus Soter Epiphanes and an Amazon on the reverse must not be linked to the victory won by Eumenes II over the Gialatians in 166 . These currency, minted to be used during panegheis. celebrated a saving epiphany of \%eus Soter. perhaps during the war between Prusia II and Attalus II.

À l’époque hellénistique. Clazomènes semble n’avoir frappé des tétradrachmes à types personnels qu au cours d"une brève période. au milieu du II" siècle. Ces émissions sont aujourd hui connues par trois exemplaires. Le premier exemplaire, acquis en Syrie du Nord et conservé au Cabinet des Médailles, n'était pas assez lisible pour en déterminer précisément la provenance. puisque l'ethnique était effacé (figure 1). Henri Seyrig l'a publié en 1971 sous le titre « un tétradrachme énigmatique » avec ce commentaire : " n'ayant rien trouvé de satisfaisant à proposer. nous nous décidons à publier cette simple description.
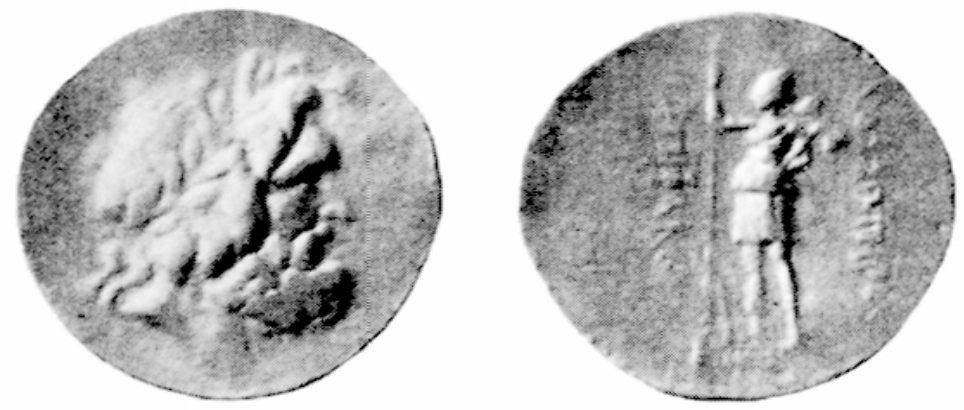

Figure 1

Lniversité François-Rabelais. 3 rue des Tanneurs. 3700) Tours - FA 4247 (¿RMAVIIA. Courriel: thibaut boulay (a wanadoofit.

1. Texte de la communication qui a été présentée lors de la journée détudes consactée à " I a monnate. source d’histoire "organisée à (Orléans le 29 févier 2008 . Je remercie frédéricue Duy rat et Catherine (irandjean de leurs conseits à cette occasion, ainsi que Séléné Psôma qui a bien voulu me transmettre son récent article sur les monnaies de pantégrie. Je remercie entin Olivier Picard pour ses remarques précieuses qui mont permis d'améliorer mon texte. Toutes les conclusions qui suivent sont. bien entendu. de ma seuke responsabilité. 
dans l'espoir que cette curieuse monnaie trouvera un exégète mieux avisé »². Georges Le Rider a attribué cette monnaie, alors unique. à Smyrne et la rangeait encore récemment parmi les tétradrachmes de cités "à types personnels » inaugurés entre 188 et 160 . comme monnaie smyrnienne. ${ }^{3}$. Le second exemplaire. plus lisible et sur lequel on distingue clairement l'ethnique clazoménien. $K \Lambda \mathrm{AZO}$. appartient à un trésor de 140 tétradrachmes découvert en Syrie en $1987^{+}$(figure 2). Cette découverte est mentionnée très rapidement par Philip Kinns dans une note de son article consacré aux ateliers monétaires d'Ionic au cours du dernier siècle de la domination achéménide ${ }^{5}$. Le trésor de Tartous a été publié en $1994^{\circ}$. mais le tétradrachme clazoménien n'a pas fait l'objet d'une étude particulière. malgré son intérêt. Enfin. un troisième exemplaire. très bien conservé. a été mis en vente le 11 janvier 2006 à New York ${ }^{7}$. S il a été brièvement analysé dans le catalogue de vente. ce tétradrachme n'a pas fait l'objet d'une autre publication (figure 3). Cet exemplaire se distingue du précédent uniquement par l’absence de marque de contrôle. Acquise par l’Alpha Bank d'Athènes'. cette monnaie a été exposée en 2007 au Bénaki Museum".
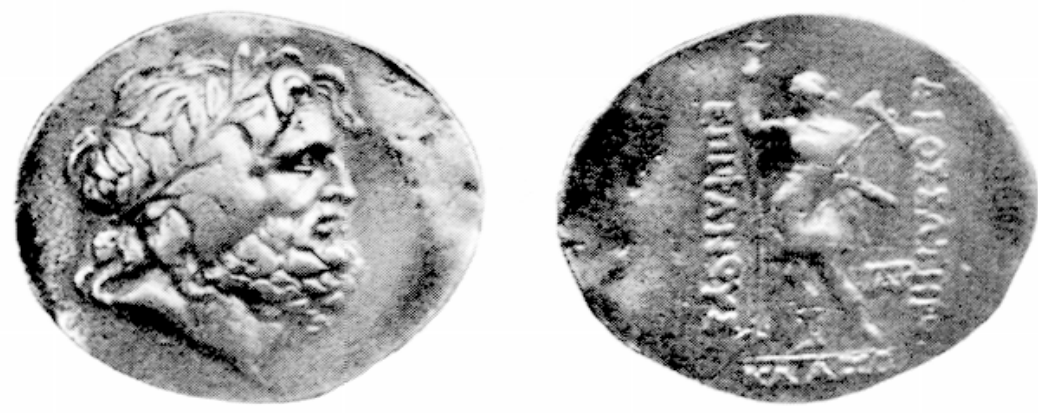

Fïgure 2

2. H. Sirrkg. Monnaies hellénistiques, XXI. Un tétradrachme énigmaticue. RN 1971. p. 24 25 et fig. 1 .

3. (i. I.: Ridt:R. Sur un aspect du comportement monétaire des villes libres d'A sie Mineure

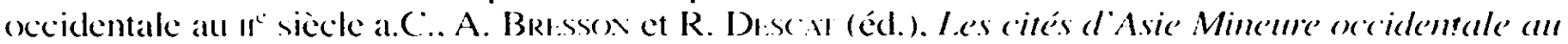
It siéle a.C. Textés réunis. Bordeaux. 2001. p. 37-63. en particulier p. $41-42$. Voir également id.. Un tétradrachme d'Athéna Niképhoros. $R N$ 1973. p. 66-79. en particulier p. 76 et 78 et lig. 10.

4. coin Hourds VIII. 471.

5. Ph. Kavs. Ioniat The Pattern of Coinage During the I alst Century of the Persian Empire. REA 91 (1989). p. 185.11. 15.

o. Coin Irourds VIII. pl. Ixiv. 1.

7. (jemini II. I.I.C (vente n" 2. lot 542).

8. Inv. Nr. 5127 : diametre: $33.5 \mathrm{~mm}:$ axe $12: 00$.

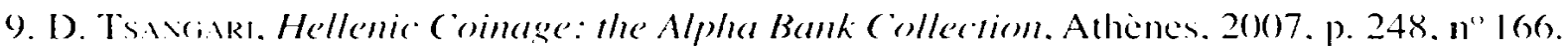
cette exposition, inaugurée le 6 juin 2007. présentait pour la premiere fois au public une partic de la collection de la banque. sur le theme de l'histoire de la monnaie grecgue. depuis son invention

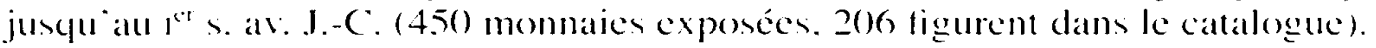




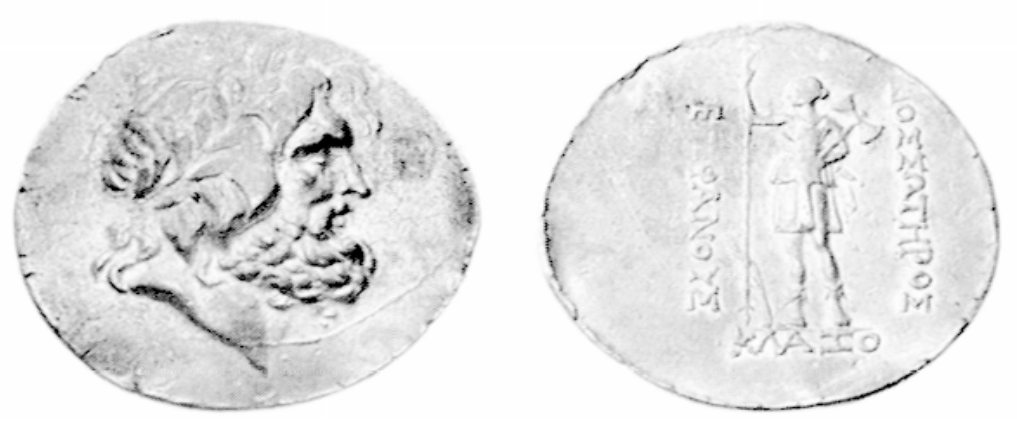

Iignure 3

Ces trois tétradrachmes sont frappés selon l'étalon attique. I e premier exemplaire. asse\% usé. a un poids de 16.48 g. le dernier exemplaire. qui semble avoir beaucoup moins circulé. a un poids de 16.69 g." Au droit figure un buste de \%eus à droite. couronné d'une couronne de laurier. Au revers est représentée une figure féminine debout. tournée vers la gauche. vêtue d'une tunique courte et de bottes. La divinité tient de la main droite une longue lance ou un sceptre. et une double hache (la bipenne) de la main gauche. appuyée contre son épaule. Elle semble porter au côté gauche une épée courte. À ses pieds à gauche. se trouve une branche de palmier. De chaque côté de cette divinité figure la légende de cette monnaie. $\triangle I O \Sigma \Sigma \Omega 2 T H P O \Sigma$ EПIФANOY $\Sigma$ K $\triangle A Z O$. C"est-à-dire le nom de Zeus Sôter Épiphanès au génitif accompagné de l'ethnique clazoménien

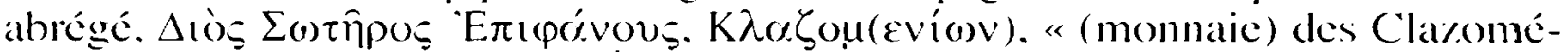
niens: (image) de \%eus Sôter Épiphanès " $"$.

La composition du trésor de Tartous ne permet guère d avancer des hypothèses concernant la date de ces émissions. Sur les 140 tétradrachmes qüil contenait. on dénombre 94 alexandres (dont 9.3 posthumes), 11 tétradrachmes émis par des cités libres $(6$ d'Athènes. 2 de Smyrne. et trois autres de Mytilène. By/ance el Clazomènes). I tétradrachme de Diodote ${ }^{\text {tr }}$ et 34 tétradrachmes séleucides, frappés entre la fin des années 150 (règne d'Alexandre I" Balas) et les années 120) (règne conjoint de Cléopâtre Théa et Antiochos VIII)'2. On peut rapprocher ces tétradrachmes clazoméniens des monnaies émises dans les années 160-140 par les cités libres voisines d’Éolide (eomme Kymè ou Myrina)

10. (omme l’indique l’intentaire de la collection. I e catalogeve de vente (sup)ra. n. 7) indiguait $10.7 .3=$

11. Olivier Picard me tait remarquer que la traduction " (monnaie) de \%eus Sôter Épiphanes.

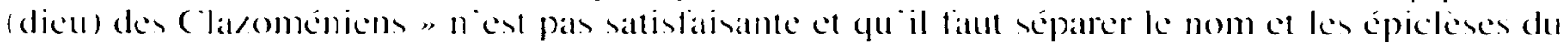
dicu. qui n apparainent que tardiement sur ke momnay ate ed lethnique abrégé ou complet yui en de regle depuis le

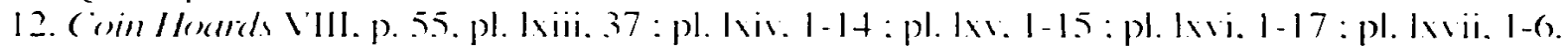


ou d’Ionie (comme Iébédos ou Magnésie du Méandre), qui sont caractérisées par l'emploi de l'étalon attique'.3. Toutefois, ces tétradrachmes de Clazomènes ne rentrent pas dans la famille des tétradrachmes avec une couronne.

Dans le catalogue de la vente de janvier 2006. Catharine I_orber a esquissé quelques hypothèses concernant les circonstances de cette émission. Rapprochant ce tétradrachme du momnayage au nom d'Athéna Nikèphoros, qu'elle rattache à la victoire d'Eumène II sur les Galates en 166 et à la "neuvième célébration du concours stéphanite des Niképhoria ${ }^{1+}$. elle propose de voir dans ces monnaies la commémoration. par Clazomènes, de cette victoire attalide qui soulagea les cités ioniennes. Les Clazoméniens auraient institué une panégyrie sur le modèle de la panégyrie instaurée à Sardes après la victoire du roi ${ }^{15}$.

Cette hypothèse soulève plusicurs problèmes. Tout d'abord, il n'y a aucun lien attesté entre la victoire d'Eumène II et la frappe d'un monnayage au nom d'Athéna Nikèphoros. Cette hypothèse repose sur la datation du décret pergaménien pour la prêtresse d'Athéna Nikèphoros, Mètris. Or, il est admis depuis 1974 et l'étude de Christopher Jones consacrée à la périodicité des Niképhoria que ce décret date de 149 et non de $165^{\circ \prime}$. Les "grands sucè̀s » fêtés à cette oecasion sont ceux remportés par Attale II contre Prusias II et non ceux d'Eumène II contre les Galates. Par ailleurs, le comportement de Clazomènes, cité libre, doit être comparé au comportement d'autres cités libres en la matière, et non au comportement d'une cité sujette et occupée, comme Sardes. Or les panégyries instaurées par les cités libres d'Asie Mineure au $\|^{\circ}$ s. célébraient toutes des divinités poliades. non des victoires royales.

Au revers de ces tétradrachmes clazoméniens figurent à la fois le nom d"une divinité all génitif et l'ethnique (abrégé). Ces monnaies doivent être rapprochées de cing monnayages, en tétradrachmes d'argent de poids attique. datant du II" siècle ${ }^{17}$.

1) Thasos (au droit la tête juvénile de Dionysos, couronné de lierre et au revers Héraklès debout, tourné vers la gauche. avec une massue et la léontè.

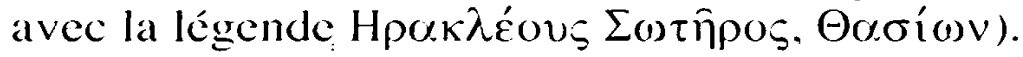

13. (j. L. Rubl:k. Sur un aspect du comportement monétaire des villes libres d'Asie Mineure

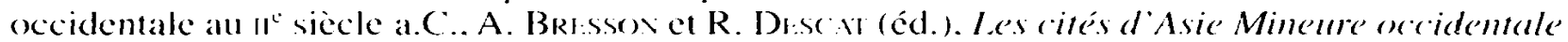
all ir siécle a.c'. Textes rélmis. Bordeaux. 2(0)1. p. 56-58.

14. $0($ is 299.1 . 1.5-16.

15. OG/S 305 .

16. Ch. P. Jovis. Diodoros Pasparos and the Nikephoria of Pergamon. (hiron 4 (1974). p. 18.3-205 (périodicité pentétérique), suivi par J. et I. RoB1:RT. Bull. 1974. 466. I. RoBk:RT. Notes d'épigraphic hellénistique. $B(H 54$ (1930), p. 3.37 (= OMS I. p. 156). avait auparavant mis en rapport les "grands succès " remportés par le roi avec le succès d'Fumène contre les Galates (166) et daté le décret pour Métris $10($ CilS 299$)$ de 16.5.

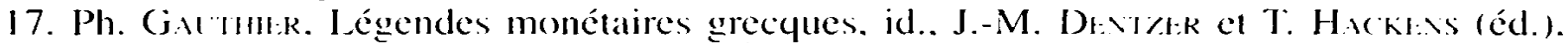
Numismatique cmique : moblèmes et méthodes. Etudes d'archéologie classique. 4. Nancy-I douvain. 1975. p. 172-173. 
2) Maronée (au droit la tête juvénile de Dionysos et au revers Dionysos debout. tenant de la main droite une grappe de raisin et deux javelines de la

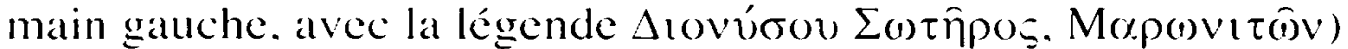

3) Alexandrie de Troade (au droit la tête laurée d Apollon et au revers Apollon Smintheus. tourné vers la droite. avec carquois, are et flèche. avec la légende

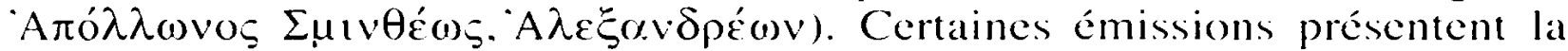
particularité d'être précisément datées"s.

4) Parion a frappé. à partir de ca 165. des tétradrachmes figurant au droit la tête d'Apollon Aktaios et au revers Apollon debout, avec patère el cithare. entre un monogramme (ou un autel enflammé ?) et l'omphalos, avec la légende

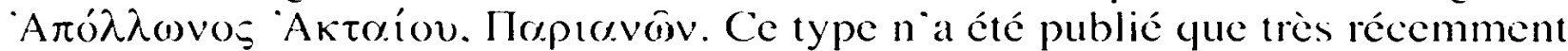
par Andrew Meadows ${ }^{19}$. La cité a ensuite frappé des tétradrachmes avec le même revers et la même légende. en ajoutant le nom d'un monétaire et en faisant figurer au droit la tête de Déméter voiléc. couronnée d’épis de blé. I a frappe, par Parion. de tétradrachmes figurant Apollon Aktaios, divinité tutélaire de sa voisine Adrastéia ${ }^{20}$. pourrait être mise en rapport avec $1^{\circ}$ annexion de cette petite cité par Parion ou témoigner des velléités d'expansion de Parion ${ }^{21}$. Antandros fit figurer dès le ${ }^{*}$ s. Artémis Astyrènè sur ses monnaies avant même d’annexer Astyra². Ce processus d assimilation des cultes des cités déchues est connu par ailleurs. Lorsque Myrina absorba Grynéion, qui possédait un sanctuaire oraculaire d'Apollon, la cité accorda une place de choix au dieu dans son panthéon civique. Apollon apparaît même sur les premiers tétradrachmes de Myrina avec une

18. La plus ancienne est datée de l'an 130 de l'ère civique (PA). soit 171/70) rexemplaire vendu le 11 mai 2005. Numismatica Ars Classicat. Auction 29. Iot 198). Ionis Robr:RT. Mommaies antiglees en Troade. Paris. p. 42. suivi par Harold B. MvrTseils. The Beginning of Athenian New Style Silver Coinage. $N\left({ }^{\circ} 1990\right.$. p. 70), pensatit qu Alexandrie de Troade avat inauguré ses frappes de tétradrachmes au type d’Apollon Smintheus à l’imitation du monnayage de la confédération d'Athéna Ilias. Dans cette hypothèse. 172/1 constitue un terminurs anté quem pour le début du monnayage de la confédération.

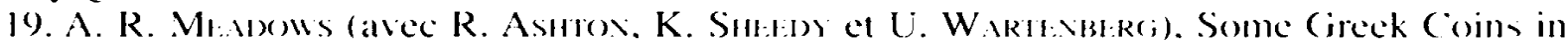
the British Museum. N( 1998. p. 37-51 et pl. 15. 10-13. en particulier p. 42-46 (la question de la datation de ces émissions doit être reprise sur de nouveaux frais. voir note précédente).

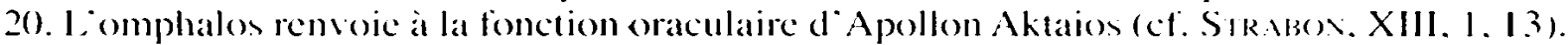

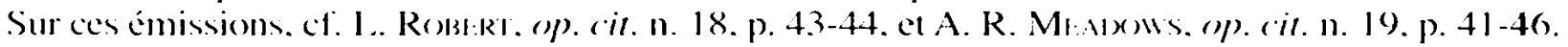

21. Adrastéiat sittéc à l’intérieur des terres. entre Parion et Priapos. conserva son autonomie. mais fut. elle aussi. victime des ag̣issements de Parion. dont elle était séparée par des collines all nord-ouest. Strabon (XIII. 1. 13) rapporte que les Pariens semparerent de blocs de marbre dans le sanctuatre de Vémésis. qui appartenait aux Adrastéiens et avait été détruit. pour construire le fameux autel élaboré par Hermokréon consacré à Apollon Aktaios qui fit la fierté des Pariens

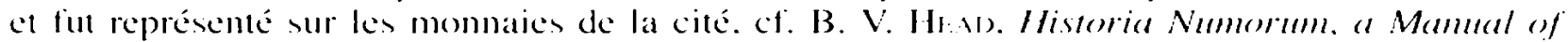
(irecek Numismatics. Oxford. 1911. p. 544. et I. Rolst:RI. op. (it. n. 18. p. 531-5.32. Sur cet atutel.

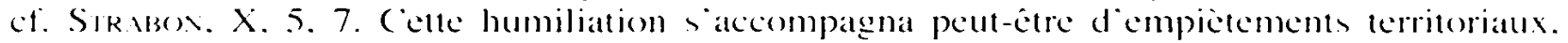

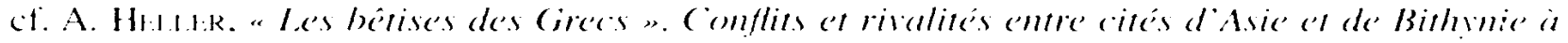

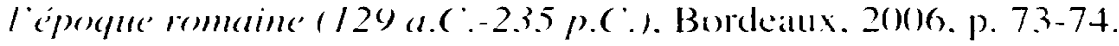

22. B. V. H(t.) (o). (it. n. 21 . p. $5+1$. 


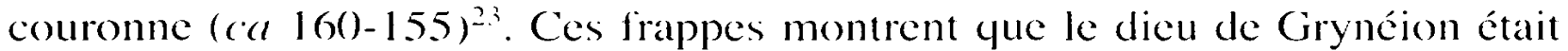
devenu l"une des divinités principales du corps civique élargi.

5) Odessos enfin (au droit la tête du « grand dieu d'Odessos » barbu, avec une bandelette dans la chevelure et au revers une divinité debout. tenant corne

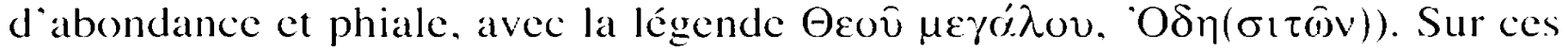
monnaies. comme sur les tétradrachmes à types personnels de Claromènes. l'ethnique est parfois abrégé.

Comme le note Philippe Gauthier à propos des cing monnayages, il faut conclure "que ees monnaies étaient des monnaies civiques, émises sous le contrôle des magistrats de la cité "2+. Il ajoute que " dans ees conditions. l'inscription du nom de la divinité avait soit une valeur honorifique (la cité. en inscrivant son nom à côté de son image. concourt au prestige du dieu au-dehors). soit une valeur religieuse (si ces émissions sont faites à l'occasion des panégyries), soit enfin (et peut-être surtout) une valeur économique et politique (si le trésor du dieu est utilisé pour la frappe des monnaies) ». On dispose à présent de suffisamment d'éléments pour tenter l'exégèse que se refusait à faire Henri Seyrig.

Il faut d'abord dire quelques mots de Zeus Sôter. Ce dieu était particulièrement invoqué «à l’approche des dangers comme à l'heure de la délivrance ou de la paix retrouvée »5. À Colophon. cité voisine de Clazomènes, lors de la réunion ( $\sigma u v \tau \varepsilon i \chi 1 \sigma u o s)$ de la vieille ville et de la ville haute (entre 311 et 306 ). pour laquelle fut organisée une importante souscription. il fut décidé de faire un vou à tous les dieux. el en premier lieu à Zeus Sôter : "que le prêtre d"Apollon. les autres prêtres et prêtresses. le prytane avec le Conseil et les personnes désignées dans ce décret. descendant sur l’ancienne agora. le 4 du mois qui commence près des autels des dieux que nos ancêtres nous ont légués. fassent le voru à Zeus Sôter. à Poséidon Asphaléios. à Apollon Claros, à la Mèter Antaia. à Athéna Polias. à tous les autres dieux et déesses et aux héros qui tiemnent notre cité et notre territoire. une fois les bienfaits accomplis, de faire une procession et un sacrifice conformément à ce qu`il plaira au peuple ${ }^{26}$. La décision prise en 196 par les néoi de Xanthos d'ériger dans leur gymnase un autel de Zeus Sôter réservé à leur gymnasiarque Lysôn. qui avait restauré ou reconstruit le bâtiment ${ }^{27}$.

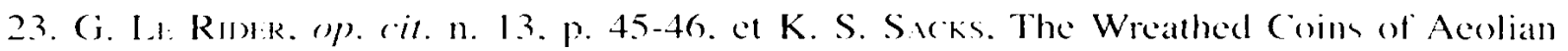
Myrhinat. ANSMN 3() (1985). p. 1-4.3.

24. Ph. (i) THIt:R. (p). (it. n. 17. p. 173.

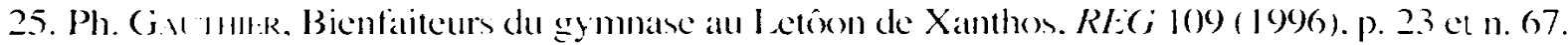

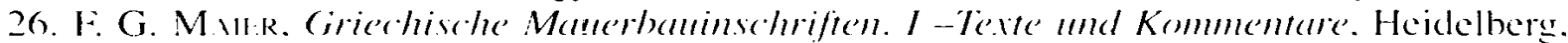

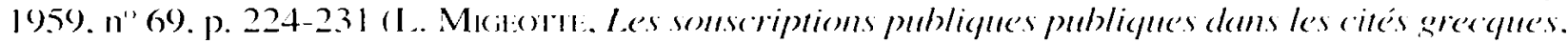
(jenere-Québec. 1992. p. 214-223. n"69). 1. 11 -21 (traduction de 1. . Migeotte).

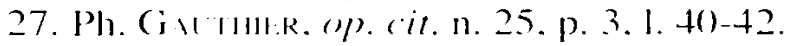


pourrait être liée aux épreuves surmontées peu de temps auparavant par le peuple. le siège de la cité par l’armée séleucide. l'année précédente. et d'éventuelles destructions ${ }^{2 x}$.

I es Grees avaient pleine eonfiance dans l’intervention de leurs dieux pour les tirer du malheur" ${ }^{29}$. On croyait à l'intervention de la divinité poliade " dans les moments cruciaux $"$ ". où la survie du corps civique était en jeu". Cette croyance venait opportunément renforcer la cohésion civique au moment où elle en avait le plus besoin. En 20)1 les Cnidiens, assiégés par Philippe V. reçurent le concours d'Artémis Hyakinthotrophos. et échappèrent au sort réservé à Kios ou Thasos". La déesse gagna une épiclèse et devint Artémis Iokuveotpópos koi Emıpovís. Lors de la guerre d'Aristonikos. Bargylia fut secourue par Artémis Kindyas. En 41. Zeus Panamaros sauva les habitants du sanctuaire de Panamara d une attaque des soldats de Labienus ${ }^{33}$. Après les guerres. les habitants des cités qui avaient reçu le secours de leurs divinités poliades ou de leurs ancêtres divins, se rassemblaient pour célébrer ces interventions salvatrices. Après l’épiphanie d’Artémis Hyakinthotrophos lors du siège de Cunide par Philippe V (?). Cnide instaura très rapidement un concours pentétérique musical et gymnique panhellénique reconnu ioorú⿴囗os par Delphes et obtint la reconnaissance de l’inviolabilité de la cité ${ }^{i t}$. Les Pergaméniens, pour remercier leur dieu

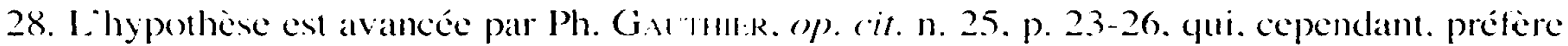
voir dans les sacrilices de l yson a \%eus Soter. l invocation du e dieu protecteur. "conservateur» du lieu lle gymnasel et de ses usagers". Sur le siege de Xanthos par l'arméc d'Antiochos III.

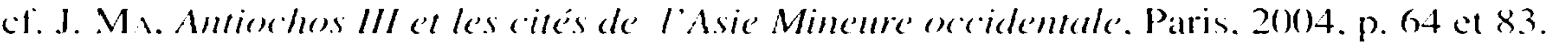

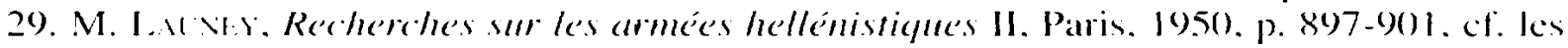

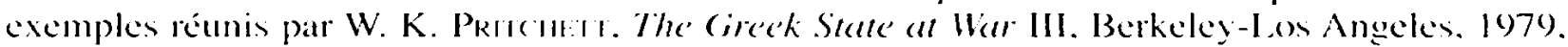
p. $29-44$.

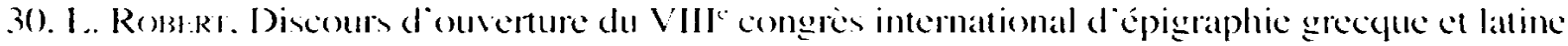

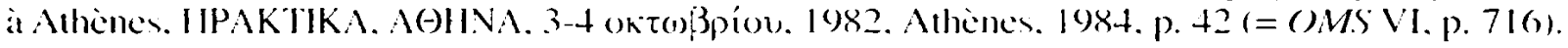

31. I es divinités continuatent également à se manifester atos côtés des troupes. En lon. Athena vint atu secours. A A

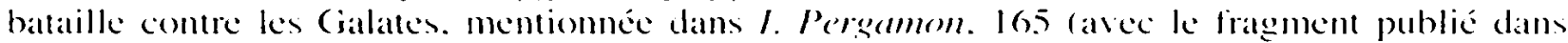

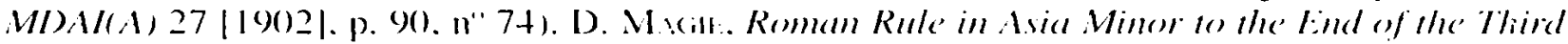

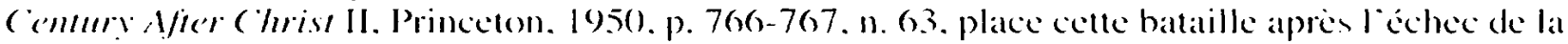

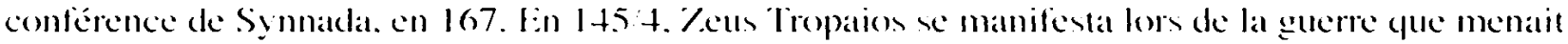

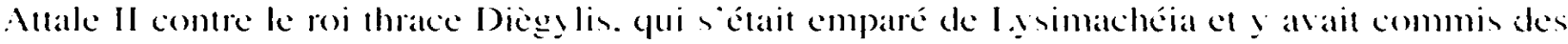
atrocités (1)

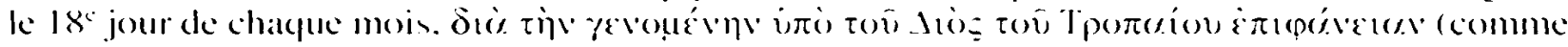

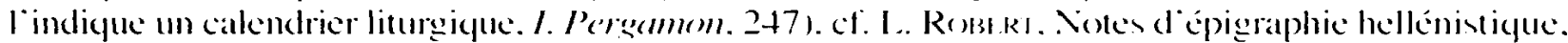

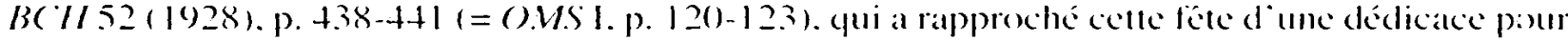
Yeus Tropatom décomente en Thrace a Bise.

32. (1. .1. I. II V.r. op. (it. 11. 29. p. 899.

3.3. I. Siranonikeial 10 .

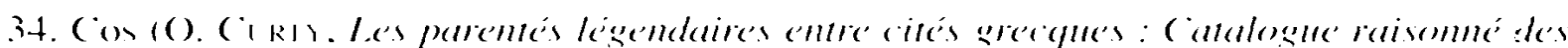

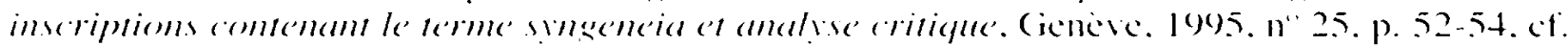

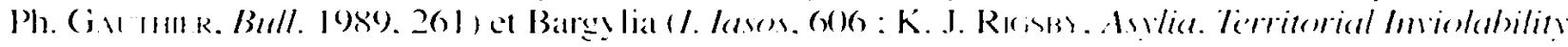


sauveur Héraklès, qui. en 155, avait repoussé l'armée de Prusias 11 , décidèrent d'annoncer ou d'instituer une fête en son honneur (associé à un autre dieu sauveur. Asklépios Sôter), les Hérakleia et les Sôtèria dès 153. Les souverains attalides. qui dès Philétairos assuraient eux aussi avoir une filiation divine avec Héraklès ${ }^{35}$. trouvèrent là le moyen de resserrer leurs liens avec les habitants de Pergame. après une guerre difficile et éprouvante, et bien sûr de célébrer leur dynastie. une nouvelle fois favorisée par la fortune. Au lendemain de l'épiphanie d'Hécate. lors de la guerre de I abienus. en 40-39, les Stratonicéens instituèrent une grande

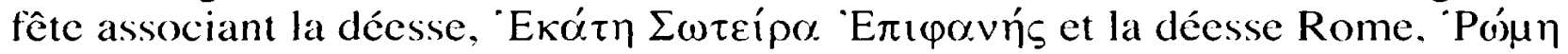

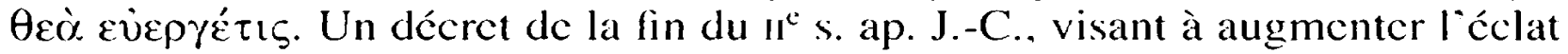
du culte d'Hécate et de Zeus, rappelait que la cité avait été sauvée, dans les nombreux, grands et continuels dangers, $\dot{\varepsilon} \kappa \pi 0 \lambda \lambda \hat{\omega} v \kappa \alpha i \mu \varepsilon \gamma \alpha \dot{\lambda} \lambda \omega \nu \kappa \alpha i \sigma v v \varepsilon \chi \hat{\omega} v$ $\kappa \iota \nu \delta u ́ v \omega \nu \sigma \varepsilon \sigma \hat{\omega} \sigma \theta \alpha \iota$, c'est-à-dire la guerre de Mithridate et la guerre contre les Parthes, grâce aux interventions de Zeus et d'Hécate, qui ont fait des miracles

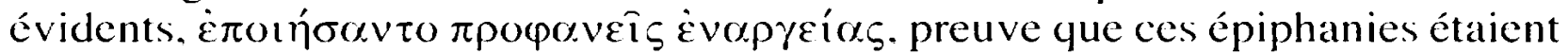
encore célébrées par la communauté civique ${ }^{36}$.

La frappe de tétradrachmes portant le nom au génitif de Zeus Sôter Épiphanès et l'ethnique (abrégé) de la cité est sans nul doute liée à l'existence d'une panégyrie locale en son honneur sur le territoire même de Clazomènes. Je crois que cette panégyrie fut instituée à la suite de l'épiphanie du dieu dans des circonstances critiques. L’association des deux épiclèses, sôter. sauveur. el épiphanès. manifeste. ne laisse guère de doute. Zeus Sôter a reçu l’épiclèse épiphanès après une intervention salvatrice. comme Hécate à Lagina et Artémis à Cnide. Ces monnaies clazoméniennes sont les seuls exemples, à ma connaissance, de l'emploi sur une monnaie de l'épiclèse épiphanès pour une divinité. En revanche. l'épiclèse figure sur le monnayage de nombreux rois de l’époque hellénistique. comme les rois séleucides Antiochos IV. Antiochos VI. Antiochos VIII. Séleucos VI, Philippe I'r , les rois de Bithynie Nicomède II ou Nicomède III. Ie roi de Paphlagonie Attale, ainsi que nombre de rois parthes. Dans ces cas, le sens est bien évidemment différent.

in the Hellenistic World. Berkeley-Los Angeles-1.ondres. 1996. n"174: O. (ikTY. (p). (it.. n" 71. p. 175-177) répondirent favorablement a linvitation des Cnidiens. ct. K. J. Rıcsis. A Hellanistic Inscription from Bargylia. (jRBS 16 (1975), p. 403-409) (J. et L. RomikT. Bull. 1976. 654) !omtra

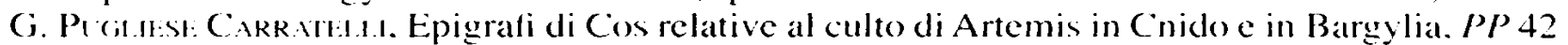
(1987). p. 119-123. Voir également le décret décidant l'envoi de trois ambassadeurs dans less cités "alliées et amies" au sujet des Hyakinthotropheia (I. Knidos, 220). I. Rori:RI. Hellenica VII. Paris. 1949. p. 116. note qu" il est possible que le prestigge de cette fête nait pas été de longue durée et qu elle n'ait pats attiré longtemps des concurrents étrangers" ».

35. L. Robi:Ri. Héraclès à Pergaine et une épigramme de l'Anthologie XVI. 91. RPh 58 (1984), p. 12-16 (= OMS VI. p. 462-466).

36. F. Sokonowski, Lois sacrées de l'Asie Mineme. Paris. 1955. n" 69. p. 162-16.5: I. Stralonikeia. 1101. cf. I. Romikt. Etudes anatoliennes. Paris. 1937. p. 516-52.3. 
On ne trouve nulle trace, dans les sources littéraires et épigraphiques. d’une éventuelle agression étrangère contre le territoire de Clazomènes au milieu du $I I^{\circ}$ s.. au cours de laquelle la cité aurait reçu le secours d'une divinité. On pourrait envisager une intervention salvatrice de Zeus Sôter au cours de la guerre qui opposa Clazomènes à Temnos, mais on voit que dans tous les cas évoqués sıpra, dieux et déesses intervenaient pour secourir une cité attaquée par un roi, comme Philippe V, Mithridate ou Eumène IH-Aristonicos, ou bien une armée étrangère. comme celle de Labienus. non pour défendre une cité de l'attaque d'une autre armée civique. Il faut done trouver la trace d'une campagne militaire menée par une armée royale ou une armée étrangère ayant touché le territoire de Clazomènes.

En 155, lors du conflit entre Prusias II et Attale II, l'armée bithynienne, qui avait tenté sans succès de soumettre Pergame puis Élaia, ravagea le territoire des cités qui se trouvaient sur sa route : les troupes de Prusias longèrent la côte éolienne et dévastèrent les territoires d’Aigai et Kymè et gagnèrent la basse vallée de l'Hermos, où elles ravagèrent le territoire d'Héraclée du Sipyle, avant de remonter la vallée de l'Hermos ${ }^{37}$. Polybe revient en détail sur cette campagne $^{3 x}$. L’armée bithynienne, après le siège de Pergame, profana les deux grands sanctuaires de la chôra pergaménienne. le Nikèphorion et l'Asklèpiéon. L a armée de Prusias II attaqua ensuite Élaia, défendue par Sôsandros sans réussir à s'emparer de cette cité portuaire ${ }^{39}$. C'est probablement après cette attaque que l'armée bithynienne pilla la pérée de Méthymna ${ }^{40}$. Puis l'armée se dirigea vers la Phrygie en ravageant le territoire des cités qui se trouvaient sur sa route. Les troupes de Prusias longèrent la côte éolienne, contournant le massif de l'Aspordènos par le sud. Les soldats traversèrent et pillèrent les territoires d'Aigai et de Kymè. Ils gagnèrent ensuite la basse vallée de l'Hermos, où elles ravagèrent sans doute le territoire d'Héraclée du Sipyle ${ }^{41}$. Sur le territoire de

37. Sur cet épisode. cif. L. RoblikT. op. cit. n. 36. p. 111-118.

38. Sur cette guerre. cf. E. WII. . Histoire politique du monde hellénistique II. Nancy. 1982. p. $381-382$.

39. PoIYB1:. XXXII, 15. 10.

40. 1. Ro13t:RT. (p). (it. n. 36. p. 114. n. 1.

41. I. RoBt:RT. ibid.. p. 112-116. propose de reconnaître Héraclée du Sipyle, près de Kymè. D). Marilt. opp. ( it. n. 31. p. 1198 . n. 43. revient à l'identification traditionnelle d Héraclée du Pont. II est suivi par F. W. W al bavk. A Historical Commentary on Polshius III. Commentary on Books XIX-XI. Oxford. 1979, p. 556. et A. DPHa cot RT. Héraclée du Pont dans les Alexipharmaca de Vicandre de Colophon : un nouvel indice de chronologie. B. VIroillos (éd.). Sutudi lillenistici XIII. Pise-Rome. 2001. p. 191-198. qui pense que Nicandre de Colophon fait allusion à ces ratages commis par te roi de Bithynie dans un passage des Alexipharmaca. v. 12-15. quand il évoque la

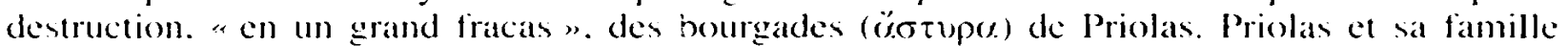
auraient "convoyé une image d"ennemis des Bithyniens" dans la littérature hellénistique (p. 198). Il faut sans doute abandonner celte hypothese. H. Fravkit. Noten all den Argonatutike 
Temnos, qui domine la rive droite de l’Hermos, Polybe indique que Prusias saccagea "l'enceinte consacrée à A pollon Kynneios. près de Temnos. et. qui plus

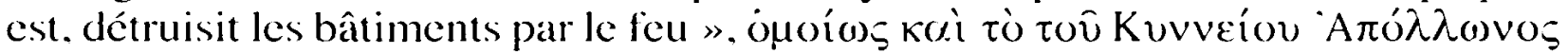

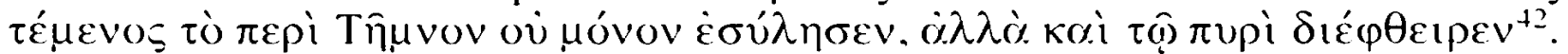
L'armée bithynienne remonta ensuite la vallée de l'Hermos et gagna la plaine hyrcanienne jusqu à Thyatire ${ }^{43}$. Les exactions se poursuivirent puisque sur le chemin du retour ( $\dot{\varepsilon} \pi \alpha^{\prime} v \delta \delta \varsigma$ ). l'armée bithynienne pilla avec violence le sanc-

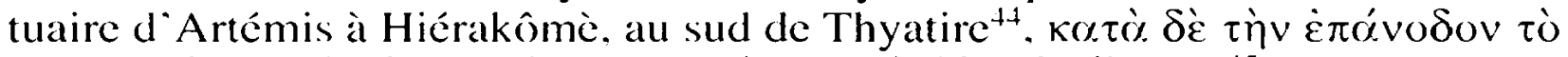

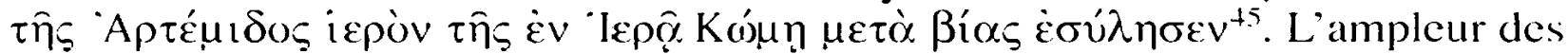
exactions commises par les soldats de Prusias fut considérable : après la guerre, Prusias fut contraint par le Sénat de verser une indemnité de 100 talents pour " la ruine ( $\kappa \alpha \tau \alpha \varphi \theta 0 \rho \alpha ́$ ) des territoires des Méthymniens, des Aigaiens, des

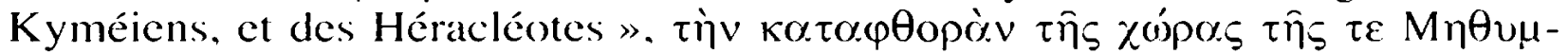

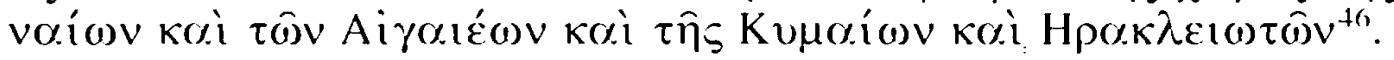

Polybe ne mentionne pas Clazomènes parmi les cités indemnisées par le roi de Bithynie. Pourtant. Clazomènes possédait une enclave territoriale dans la basse vallée de l'Hermos. Dans la première moitié du II" s., une guerre violente éclata entre Clazomènes et Temnos. cité éolienne située non loin de l'embouchure de l'Hermos. Un arbitrage de Cnide mit un terme à leur affrontement ${ }^{47}$. L'une des accusations portées contre les Clazoméniens concernait la manipulation des bornes d'un téménos. peut-être sur un territoire que les Temnitains revendiquaient comme leur. En l'absence de témoignage fiable. les Cnidiens innocentèrent les Clazoméniens. Comme l’a bien vu l’éditeur de cet arbitrage cnidien. Peter Herrmann. ceux-ci possédaient donc une «pérée » en Éolide. vers l'embouchure de $l^{\prime} \mathrm{Hermos}^{+\mathrm{t}}$. Cette possession remonte au moins au début

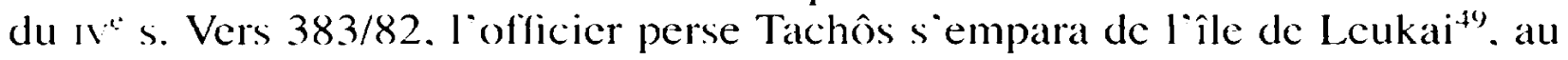

des Apollonios. Munich. 1968. p. 232. n. 218. suggérait qu Apollonios de Rhodes. 1. 774-810. faisait allusion aux conflits de Lysimaque et d'Arsinoè contre les Bithyniens (A. D'HACOR R. ibid., p. 198, n. 29).

42. PoI.YBt: XXXII. 15. 12.

43. Pol. YB1:. XXXII. 15. 10.

44. I a future Hiérokaisareia. Sur les différentes Hiérakômè. ef. L. Robi:RT. op. cit. n. 36. p. $559-560$.

45. Po1, X1.. XXXII. 15.11.

46. Pol.YB1.. XXXIII. 13. 8. Cette liste n'est pas exhaustive, puisque n'y figure aucune cité lydienne ni même Temnos. pourtant touchée par les ravages commis par les soldats de Prusias II. On ne peut donc tirer atcune conclusion ferme de labsence de Clazomenes parmi les cités indemnisées.

47. P. HI:RRMIW. Die Stadt Temmos und ihre auswartligen Beyichungen in hellenistischer \%eit. MIDA/(I) 29 (1979), p. 249-271 (.SE(; 29.1130). cf. J. et I.. RoBt:RT. Bull. 1980. 438) : S. I.. Aril.R. Interstate Arbitrations in the (ireek world: 3.37-90 B.C.. Berkeley-Los Angeles. 1996. n"71. p. 186-192 (ct. remarques de Ph. Gicthit.k. Topoi 8 |1998|. p. 320).

48. P. Ht:RRMAli. op. cit, n. 47. p. 259-262.

49. Ies atterrissements de l'Hermos provoquèrent le rattachement de lîle au continent au début de l'époque romaine. eff. PtIN:. N.H. V. 119. 
sud de Phocée et au nord de Clazomènes. pour en faire sa base d'opération contre le Grand Roi ${ }^{50}$. Il décida dy fonder une citésl. À la mort de Tachôs. peu de temps après la création de Leukai. Kymè el Clazomènes se disputèrent

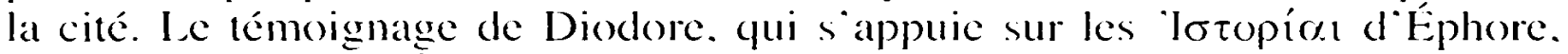
originaire de Kymè $e^{52}$. indique clairement que les Clazoméniens possédaient. dès cette époque. une enclave territoriale à l'embouchure de l Hermos ${ }^{53}$. ce qui explique leur ambition sur Leukai. Strabon rapporte que les Clazoméniens possédaient en outre un domaine maritime dans le golfe de Smyrne évoquant " huit petites îles fertiles et bien cultivées ${ }^{5.4}$. Parmi ees îles on peut relever les îles de Pélè et Marathoussa ${ }^{55}$. L ors du règlement d'Apamée. les Romains attribuèrent Drymousa ${ }^{56}$ à Smyrne. en récompense de son attitude pendant la guerre contre Antiochos III ${ }^{57}$.

I a présence cla\%oménienne en Éolide provoqua sans doute des tensions très vives avec les voisins immédiats: outre Temnos et Kymè. on peut penser à Larissa et Néonteichos. I alluvionnement du fleuve donna naissance à des

$50 . \mathrm{DI}()() \mathrm{RL}, \mathrm{XV}, 92,1-5$.

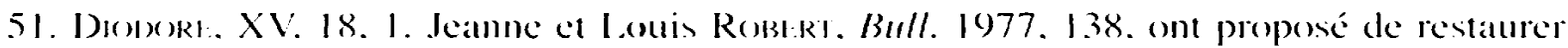

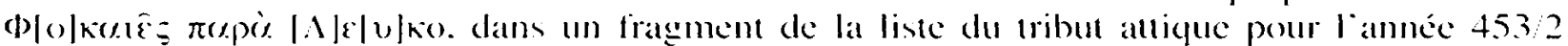

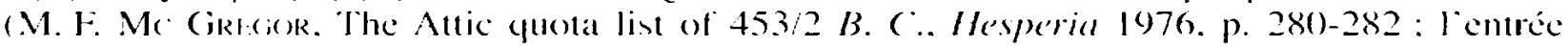

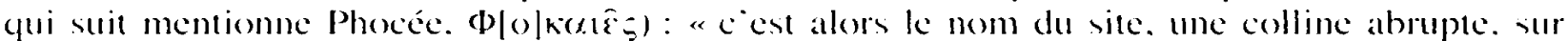

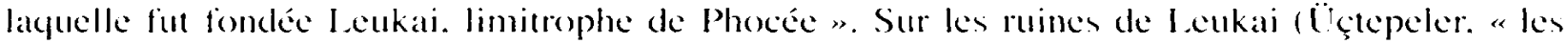

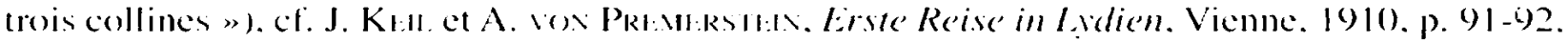

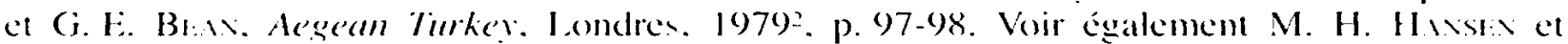

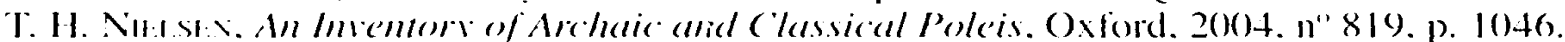

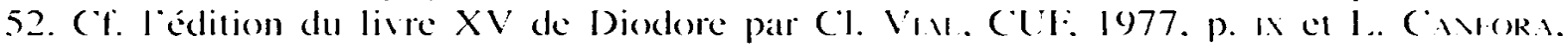

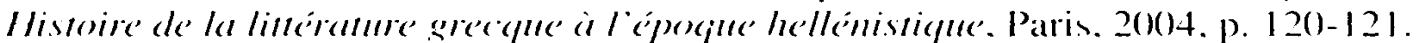

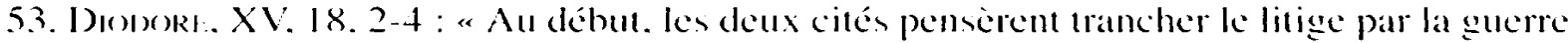

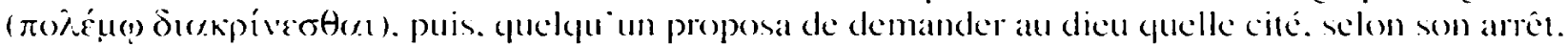
devait être la mâtresse de lecukati. I arrêt rendu par la Pythie fut que célat la cité qui offrirait lat premiere un sacrifice dans la cité de I eukai. (hakeun partiatil de sa ville, au lever du soleil. un jour

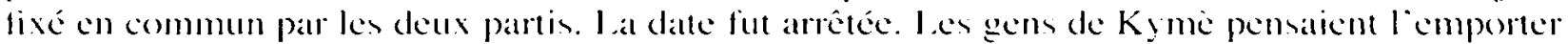
parce que leur ville était plus proche de l eukai, mais ceux de ( ladomenes. plus éloignés. sassurent la victoire par le smatagène suivant : des colons furent désignés pour fonder une cité près de I cukai : ils en partirent au lever du soleil et accomplirent le sabrifice asant les gens de Kyme.

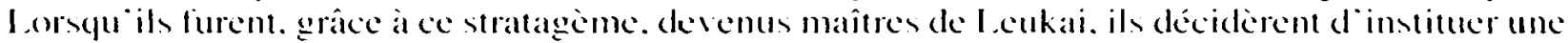

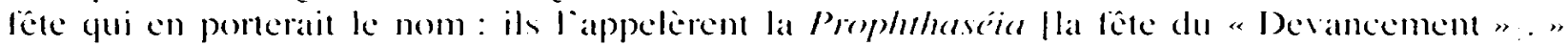

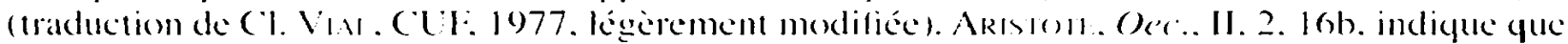
la cité recruta un corps de mercenaires. el contractat une dette de 20 talents. (ef. Ph. KNis, op). ith. 11. 5.p. $18.5-186$

54. SIR Viso XIV. 1.36

5.5. I ors de la révolte des cités ioniennes soutenue par Sparte et la Perse. les habitants de

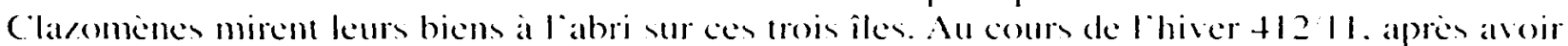
Échoué a prende la cité. le navarque spartiate Astyochos abordat sur ces îles et s"empatra des biens

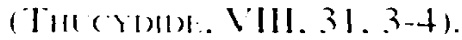

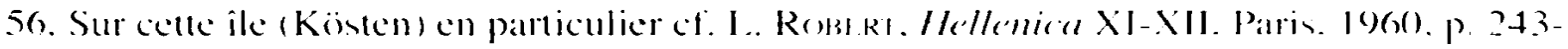

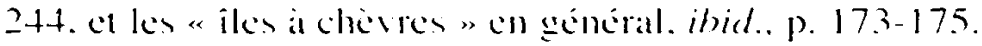

57. P() yB1. XX1. 46. 5. (1. I. RoB1R1. (p) (it. n. 56. p. 51.3-514. 
salines et de nouvelles terres fertiles, très convoitées. Malgré les témoignages épigraphiques et littéraires sur cette enclave territoriale. il n'est pas possible d'en préciser l'emplacement exact. Toutefois, on note que parmi les monnaies découvertes lors des fouilles de Larissa, les monnaies de Clazomènes étaient plus nombreuses (une d'argent. cinq de bronze) que celles de Néonteichos située à $5 \mathrm{~km}$ (deux bronzes). celles de Kymè. située à $15 \mathrm{~km}$ (trois bronzes). celles de Chios (un bronze) et celles de Téos (une d'argent) ${ }^{58}$. Même s'il ne faut pas surinterpréter ces données. il est probable que la pérée clazoménienne jouxtait à la fois les territoires de Larissa et de Temnos. Celle-ci se trouva sans doute sur la route de l'armée bithynienne. Zeus pourrait être intervenu contre ces soldats impies, qui avaient profané nombre de sanctuaires, afin de « sauver » ce territoire clazoménien.

Les considérants d'un décret de Stratonicée exposent ainsi avec force détails les prodiges de Zeus pour la sauvegarde du chôrion de Panamara et de ses habitants, attaqués par un détachement de troupes envoyé par Labienus. à la fin de l'année 41 ou au début de l’année $40^{59}$. Ce $\psi \eta ́ \varphi \imath \sigma \mu \alpha$, qui exprimait la reconnaissance du peuple envers le dieu et décidait la construction d'un monument commémoratif. fut gravé sur l "un des murs du sanctuaire (") $)^{(x)}$. Il donne le détail des différentes interventions miraculeuses du dieu. qui lança des flammes et des éclairs contre les assaillants, fit répandre un brouillard épais, déchaîna lés éléments contre eux. et envoya une meute de chiens fantômes pour accompagner leur déroute... Ce témoignage carien donne une petite idée du secours que fut en mesure d’apporter Zeus Sôter aux habitants de la pérée clazoménienne. Larbitrage enidien qui régla le conflit entre Temnos et Clazomènes évoque un sanctuaire sur la pérée clazoménienne. Celui-ci appartenait peut-être à Zeus Sôter.

Après son épiphanie. que je propose donc de dater de 155. Zeus Sôter s'est affirmé comme l'une des divinités tutélaires de Clazomènes. Cela est confirmé par l'apparition. dans le courant du $\mathrm{II}^{\circ} \mathrm{s}$. de monnaies de bronze figurant au droit Zeus"1 dans lequel on peut reconnaître désormais Zeus Sôter Épiphanès (figure 4). La représentation du dieu est similaire sur les monnaies de bronze et sur les tétradrachmes. Auparavant seuls Athéna et Apollon figuraient au droit des monnaies de la cité et Athéna devait être la divinité principale de la citér?

58. L. Rob1:R1. Eutudes de mumismatique grectue. Paris. 1951. p. 67. On releve également sept mommaies de Colophon.

59. I. Stratonikeia. 10.

60. Sur le lieu de gravure du décret. cf. P. Ros sst:1. Le miracle de Zeus Panamaros. BCH 55 (1931). p. 76-77.

61. B. V. Hexp. (1). (it. n. 21. p. 568-564.

62. $B M C$ XIV Ionia (1892), p. 2 I-28. 


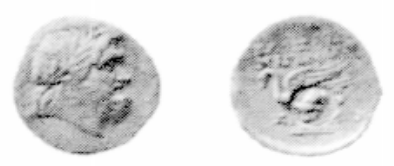

ligure 4

I épigraphie de la cité. très pauvre, ne permet pas de trouver la trace de l instauration d’une fête el d’une panégyrie en l’honneur de Zeus Sôter Épiphanès. On relève seulement la consécration. à la basse époque hellénistique. d un autel à Zeus Sôter par un affranchi. Cimnamos" : Frity Graf ${ }^{\text {rht }}$ supposait l'existence à Clazomènes. d'un culte de Zeus Olympios. car une monnaie de la cité du début du N. porte le nom théophore d'Olympiodôros $^{0.5}$. John Cook a exprimé ses réserves sur cette hypothèse de Grafro. Après la première guerre de Mithridate. à Lampsaque, Priape, puis Aphrodite. tous deux qualifiés d "Erıpovińs furent lun et l'autre prytane épony me"7. Leur épiclèse pourrait également se rapporter à des interventions dans des moments critiques au secours des habitants de Lampsaquc.

Reste une difficulté à éclaircir : la présence de la figure féminine, au revers de ces tétradrachmes clazoméniens ne manque pas de surprendre. On s"attendrait à trouver un symbole ou un attribut de Zeus. Cette figure féminine tient dans sa main droite la bipenne, qui constitue un symbole de puissance des divinités célestes et apparaît notamment entre les mains de Zeus I abraundos en Carie. Catharine Lorber identifie cette fịure féminine à Artémis. Elle suppose que la bipenne est l"attribut de Zeus Labraundos et qu“à Clazomènes le culte du dieu carien aurait été assimilé à celui de Zeus Sôter. protecteur de la liberté de la cité. J aurais tendance à voir dans cette figure une Amazone, comme le proposait déjà Henri Seyrig. suivi par Georges Le Rider. En effet. les Amazones sont souvent représentées avec une bipenne. I a présence de cette Amazone est sans doute liéc à l"histoire mythique de la cité. Les Amazones jouent un rôle signnificatif dans les légendes de fondation de plusieurs autres cités d'Asie Mincure

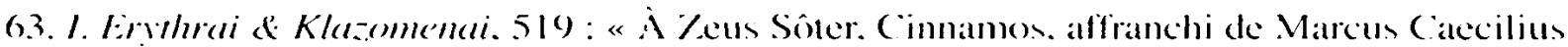

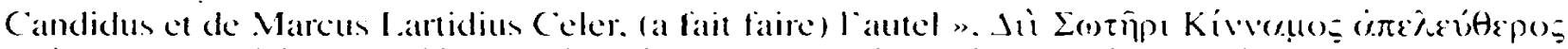

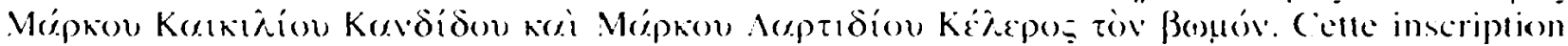

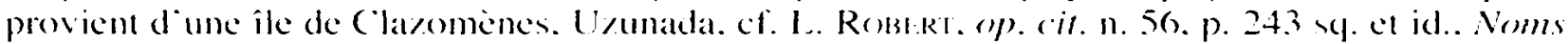

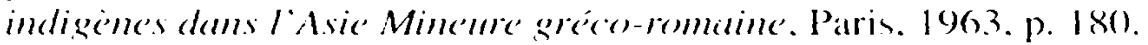

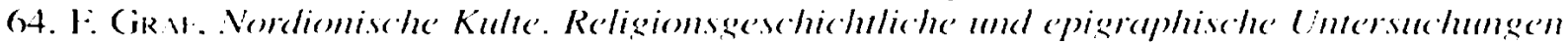

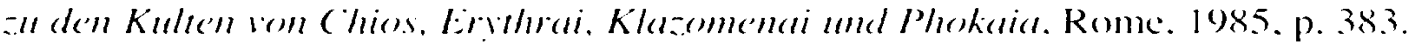

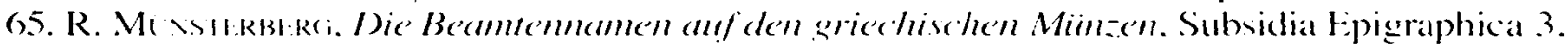
Hildesheim-New York. 197.3. p. 82.

66. I. M. (i)(x. I/IS 107 (1987). p. 219-220.

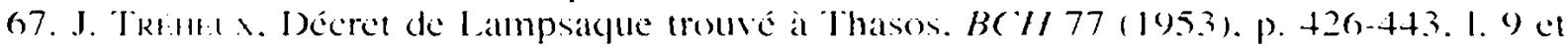
$27-28$. 
oceidentalesx. Les Amazones sétaient emparées de l'Ionie et deux voisines de Clazomènes. Kymè et Smyrne. furent fondécs par elles, tout eomme Myrina. Pitanè. Mytilène. Éphèse. Priène et peut-être Boionè ${ }^{\prime \prime}$. Strabon. qui dénonçait l'invraisemblance de ces récits concernant ces hérö̈nes et leur rôle dans la fondation de cités, notait que ces récits avaient encore cours à son époque ${ }^{70}$. La palme, au pied de l'A mazone, évoquait sans doute un événement heureux de l’histoire ancienne ou récente des Clazoméniens.

Louis Robert rappelait que le monnayage d'une cité « est un morecau de 1 histoire politique des cités $\gg 71$. Les «énigmatiques » tétradrachmes de Clazomènes, pour reprendre le mot d'Henri Seyrig. montrent une nouvelle fois. si l'on accepte les propositions que je formule ici. tout ce que l'étude d'une monnaie peut apporter à la compréhension de l’histoire locale d'une cité. en croisant les informations qu'elle livre avec ce que l'on sait par ailleurs grâce à la tradition littéraire, à l’épiggraphie ou à la géographie historique. Au cours de la campagne dévastatrice de Prusias II en Éolide (en 155). l 'armée bithynienne aurait pénétré dans l'enclave territoriale que les Clazoméniens possédaient dans la basse vallée de l'Hermos. non loin de l'embouchure du fleuve. et une épiphanie de Zeus Sôter aurait chassé les assaillants. Après cette intervention salvatrice. les Clazoméniens auraient institué une fête et une panégyrie en l`honneur du dieu². de même que les Pergaméniens avaient institué des Hérakléia après qu Héraklès eut repoussé l’armée de Prusias II. Selon Sélèné Psôma. ces monnaies frappées à l'occasion de panégyries constituaient le seul monnayage légal (dokimon nomisma pour les paiements importants durant l'événement. Par ailleurs. toutes les transactions à grande échelle devaient être effectuées avec ces momnaies.;

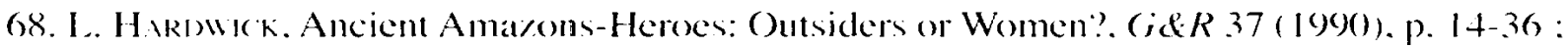
K. Dowsw. The Amazons: Developpement and Functions. RhM 140)(1907). p. 97-128.

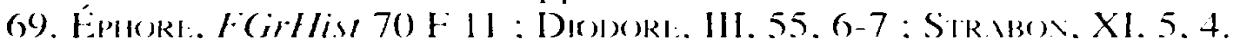

70. STR IBOS. XI, 5, 3-4.

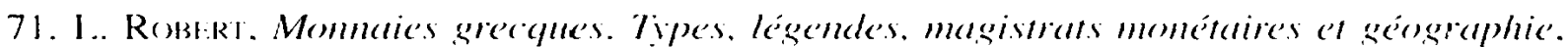
Paris-Cieneve. 1967. p. 14.

72. Récemment a été publié un tétradrachme att nom des technites dionysiacues. contemporain des émissions de Clayomines (C. C. I. okbl:R et O. D. Hox).R. An Lnpublished Tetradrachm Isstled by the Artists of Iongsos. NC 2003. p. 59-68. pl. 15. 1-2). Au droit figure Dionysos. pontant un bandeau. eouromé de lierre. Au revers on trouve la légende et le thyrse de Dionysos. dans une couronne de lierre. I es éditeurs de cette monnaie ont fondé leur interprétation sur l'hypothèse que Téos, ou sétaient installés les technites dionysiaques. était soms domination attalide au u"s. apres Apaméc. Or il nén est rien. malgré la présence. dans la cité. d’un groupe d'artiste dévoué a la dynastie attalide. les Attalistes. créé par le célebre joueur de flute Kratón. Je reviendrai ailleurs sur cette question.

7.3. S. Psom. Profitable Networks: Coinages. Panegrris and Diony siac Artists. Meditereaneem Ifistrrical Review 22 (2007). p. 237-255. en particulier p. 24.3 : "The organimers of the fairs obliged the participants by law to exchange their currencies on Attic or other weight eoinages for the currency in name and types of the honoured god, and probably to pay an agio for the procedure. " 
L 'existence de différentes émissions (l'exemplaire de l'Alpha Bank ne porte aucune marque de contrôle à la différence de l'exemplaire du trésor de Tartous) pourrait indiquer que ces frappes de « monnaies de panégyries» se sont répétées à plusieurs reprises. Par la suite. la fête en l'honneur de Zeus Sôter Épiphanès a peut-être connu le même destin que les Hérakléia de Pergame, qui cessèrent d'être célébrées au cours du ir siècle av. J.-C. ${ }^{7+}$

74. L. R(131.RI. (p). (it. n. 35. p. 17-18(= OM.S VI. p. 467-468). 